\title{
III
}

\section{THE VALUE OF SERUM REACTIONS IN THE DIAGNOSIS AND TREATMENT OF VENEREAL DISEASES *}

By T. E. OSMOND, M.B., Pathologist, V.D. Dept., St. Thomas's Hospital

Madam President, I.adies and Gentlemen,-I chose this title for my paper to-night deliberately because there is so much misunderstanding about the interpretation of these tests, and I had hoped, perhaps, to offer a little help to some of our "weaker brethren "; I regret that I see no "weaker brethren " present-but none the less I trust you will bear with me.

When I was a student-I do not care to think how many years ago-a positive Wassermann reaction meant syphilis and a negative excluded it. Now I know that no one here present believes this, but unfortunately there are still many people who do-and people who should know better. The value of any test depends on two factors - the skill and experience of the tester and the specificity and sensitivity of the test. Now I suppose there are few tests in all pathology more complicated or more capable of error than the Wassermann, yet this reaction is carried out in all sorts of laboratories all over the country, often by an unqualified attendant, and people accept their reports like a baby does its bottle. There are all sorts of ways of doing the Wassermanngood, bad and indifferent-mostly bad, and it is these which have made the Wassermann reaction one of the most abused-I use the term in two senses-of all tests. When you want a serum test done, therefore, choose a pathologist in whom you have confidence and whose technique is an approved one. It has been laid down authoritatively that a laboratory should perform at least fifty Wassermann reactions a week if its results are to be reliable. I would go further and say that it should be in touch with the clinician as well-otherwise how is the

* An address delivered before the Medical Society for the Study of Venereal Disease, March 29, I935. 


\section{BRITISH JOURNAL OF VENEREAL DISEASES}

pathologist to check his results ? One more thing-if you want the best results you must send good specimens; a hæmolysed or infected specimen only adds to the pathologist's difficulties. Rinse out your syringe with sterile saline before use-take at least ro c.c. of blood so that confirmatory tests may be done if necessary-and if you are going to post it allow the specimen to stand several hours so that serum and clot may separate before you pack it up : better still, pour off the serum and send only that. Supply clinical details-it helps the pathologist to interpret the result. Now I do not intend to confine myself to the Wassermann reaction, but propose to take that reaction first and compare it with others later. First of all, what about its specificity ? For all practical purposes a positive Wassermann reaction means syphilis, and what I say of the Wassermann reaction is largely applicable to most of the flocculation tests. (It is hardly necessary to add that an unexpected positive Wassermann reaction should be repeated on a fresh specimen to avoid the possibility of either a technical error or a mixture of specimens.) The only real exception to this is yaws-a matter of small moment to most, since it is rarely seen in the British Isles. Yaws, which is halfbrother to syphilis, presents much the same clinical picture, is caused by an organism indistinguishable from the $S p$. pallida, and reacts to arsenobenzol and bismuth. Undoubtedly sometimes leprosy gives a positive reaction. At the Serum Conference at Monte Video in I93I sera from 27 cases were examined by twelve different methods, with the result that about 30 per cent. of positives were recorded - no method being immune. Amies ${ }^{1}$ agrees and considers that the reaction may be expected to be positive in advanced cases, particularly when there is much destruction of tissue, and quotes Lloyd, Muir and Mitra ${ }^{2}$ in support. However, in this country leprosy is not likely to cause much trouble in differential diagnosis. A whole host of other conditions has been implicated at one time or another, including various febrile conditions such as relapsing, scarlet, typhus, typhoid and malarial fevers, pregnancy, diabetes, tuberculosis and malignant disease, and a number of skin conditions, notably psoriasis, pityriasis rosea and others. Of these relapsing fever is not likely to cause confusion. As regards malaria, Thomson and Mills in I9I9 got 8 positives in I30 cases, 


\section{THE VALUE OF SERUM REACTIONS}

but all were found to be suffering from concomitant syphilis-whilst I myself have tested sera in India and sent from India with the same result ; I have also tested a considerable number of sera from scarlet fever cases without recording a false positive. What does happen, in my experience, is that some of the above diseases do give $\mathrm{a} \pm$ or doubtful reaction, but not a frank positiveat any rate with the Wassermann reaction (No. I Method M.R.C.). I think it is easy to account for the long list of conditions which may or do cause a false positive Wassermann reaction which appears in so many text-books. Someone, probably using a poor technique, records a false positive in such and such a condition; others follow with other experiences and these get copied from book to book, and so the list grows. All I can say is, do not believe it! Kolmer ${ }^{3}$ says-barring yaws and perhaps relapsing fever-that the complement-fixation test is " practically specific for syphilis under acceptable technical conditions."

Another point which I should like to mention here is the use of the expression " weak positive." To my mind a complement-fixation test can only give three possible results-positive, negative and doubtful, often recorded as plus, minus, and plus over minus respectively. I do not think the term "weak positive" should be applied to a \pm result where the diagnosis is concerned, though I admit that the two terms may be synonymous in a treated case of syphilis: on the whole it is better to avoid the expression altogether. In this connection I should like to say a word about nomenclature. Surely it is best to stick to a universally understood system such as that recommended by the League of Nations, i.e., positive or + , negative or - , and doubtful or \pm ; if anyone likes to use his own pet method, by all means let him do so, but he should in addition use the word positive, negative or doubtful so that there may be no risk of misunderstanding.

As regards specificity, what applies to the Wassermann applies also to most of the better known flocculation tests, such as the Kahn, Sigma, Müller-Ballungs, MeinickeKlärungs, Kline and Hinton reactions-to mention those with which I am most familiar. Certainly in the author's own hands the Standard Kahn test showed a most remarkable degree of specificity at the Copenhagen and Monte Video Serum Conferences, only equalled by the 


\section{BRITISH JOURNAL OF VENEREAL DISEASES}

Wassermann reaction as carried out by Dr. Wyler and originally elaborated by Colonel Harrison, whose humble disciple I am. Unfortunately in the hands of others the Kahn test has not proved quite Ioo per cent. specific-a remark which applies to most of the other flocculation tests - and I confess that when it is a case of diagnosis I would put more faith in a positive Wassermann reaction (No. I Method) than in any flocculation test.

Now in what proportion of cases may we expect a serum test to be positive in the various stages of syphilis?

In primary syphilis Kolmer gives the following figures :

5-I4 days after the appearance of the sore, 44 per cent.

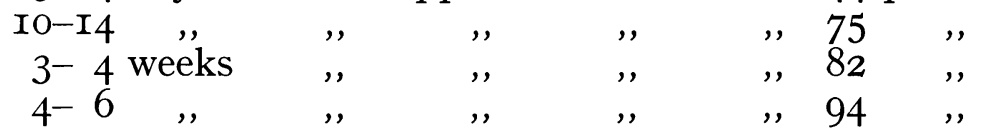

I have recently looked through the case-sheets of most of the cases of primary syphilis reporting at the St. Thomas's Hospital clinic during the last two years and found a positive reaction before the seventh day very rare. My opinion is that one may begin to expect a positive reaction any time from the seventh day on-the longer the time the greater the probability of a positive reaction. On the other hand, where a case presents a suspicious lesion in which $S p$. pallida cannot be found, frequent serum tests should be carried out for at least six weeks - say, every seven days - and at least two more at monthly intervals before one can pronounce the patient safe from infection.

In secondary syphilis the percentage of positive reactions rises practically to Ioo. In my experience it is very rare to meet a case giving anything but a strongly positive reaction-so rare that one would be tempted to revise the diagnosis in the absence of demonstrable $S p$. pallida however typical the signs, especially when it is remembered how many conditions can simulate a syphilitic rash, such as seborrhœic dermatitis, pityriasis rosea, psoriasis, lichen planus, various urticarias and erythema multiforme. Furthermore, not all condylomata are syphilitic, whilst mucous patches may be confused with Vincent's angina, diphtheria, streptococcal infection of throat and various forms of stomatitis and glossitis.

It is perhaps in the detection of so-called latent syphilis that the serum tests are most valuable. It is not rare 


\section{THE VALUE OF SERUM REACTIONS}

for a history of chancre and secondary lesions to be lacking, and the disease may only be latent in the sense that it shows no obvious external signs, whereas it may be active in concealed situations such as the cardiovascular and central nervous systems. It is not possible to give an accurate figure, but I suggest that the serum test may be positive in anything from 70 to 80 per cent. of cases. Here I would like to interpolate what I believe to be a sound principle, and that is, examine the C.S.F. of a latent syphilitic who gives a positive Wassermann reaction at the earliest moment, or at any rate as soon as you see he is not reacting to treatment-which may be judged by Sigma tests - a point which I shall refer to later.

In tertiary syphilis the percentage of positives varies with the organ or part involved. Kolmer gives the following figures :-

Skin and mucous membrane . 90-96 per cent.

Bones and joints . . . . 80-90 ,,

Gastro-intestinal . . . 90-95 ,,

Liver and spleen . . . 90-95 ,,

Cardio-vascular . . . 80-96 ",

Eye, ear, nose, throat . . 85-97 ,,

However, I think most people would not put the figures quite so high. In my experience, taking tertiary sypinilis as a whole, the percentage lies between 70 and 80 .

In neuro-syphilis in general a high percentage of positives may be expected, but it is important to remember that a negative serum reaction may be associated with a positive cerebro-spinal fluid and vice versâ.

Positives in the Blood.

Asymptomatic neuro-syphilis gives 70-Ioo per cent. Tabes dorsalis

Paresis

Cerebro-spinal syphilis

$$
\begin{array}{lll}
\text {, } & 70-85 & , \\
,, & 98-100 & , \\
,, & 80-90 & \text {, }
\end{array}
$$

I propose to refer to the cerebro-spinal fluid reactions later.

As regards congenital syphilis it is difficult to give a figure. A child born with obvious lesions practically always gives a positive reaction; on the other hand, syphilitic children born without signs may react negatively 


\section{BRITISH JOURNAL OF VENEREAL DISEASES}

at first and positively later, or vice versâ. In the absence of active signs the percentage of positive reactions tends to diminish with the lapse of time. In all cases of suspected congenital syphilis the blood of both the parents should be tested; if the mother's blood is positive and she has not been treated during pregnancy the likelihood is that the child is syphilitic; at any rate it must be kept under observation for a considerable period. In this connection the effect of pregnancy must be considered; pregnancy has been accused of causing false positives. I believe the opposite holds, that is to say, it is more liable to cause false negatives. A serum test on cord blood is often unsatisfactory as being anti-complementary -in any case I would not attach much value to itthough a positive reaction should bring both mother and child under strong suspicion.

For test of cure it must be remembered that a single negative test is not sufficient. In early syphilis a series of negative tests extending over a period of at least two years - say, four in the first year and two in the secondis the minimum necessary to establish the fact of cure. But remember this in itself is not sufficient. In addition the patient must have received a given minimum amount of treatment and must have a negative cerebro-spinal fluid, preferably taken towards the end of the second year of observation. In late syphilis the blood reactions often remain positive more or less indefinitely, a condition commonly referred to as "Wassermann-fastness." I shall refer to this again in connection with quantitative tests, but I would like to stress two points in this connection :-

(I) Patients giving a persistently positive blood reaction should have their cerebro-spinal fluids examined.

(2) Such patients should, in my opinion, be watched throughout life, and unless there are contra-indications should receive some treatment, such as a course of bismuth every year, as an insurance.

What is the cause of Wassermann-fastness we do not know, but most people believe it is an indication of the presence of living spirochætes lying hidden somewhere in the tissues, especially in view of Warthin's ${ }^{4}$ work. I doubt if any number of negative reactions is sufficient to establish cure in late syphilis ; in any case I would advise testing the patient's blood at least once a year throughout 


\section{THE VALUE OF SERUM REACTIONS}

life. If you see a case of late or latent syphilis giving a negative Wassermann reaction and you doubt whether he has had sufficient treatment, a provocative injection is worth trying, testing the blood on the third, fifth, seventh, tenth and fourteenth days following the injectionthat taken on the seventh day being perhaps the most important. If any of you doubt the value of a provocative test let him re-test all his sero-negative late primary cases a week after the first injection ; he may be surprised at the number of positive reactions.

Here let me say something about a \pm , or doubtful, serum reaction. For diagnosis it can be regarded as no more than suspicious and a stimulus to further investigation of the case both clinical and serological. In a treated case of syphilis it may be regarded as equivalent to a weak positive and as an indication for further treatment. It is not infrequently found in early untreated syphilismeaning that the reaction is gradually becoming positive -and in insufficiently treated cases indicates that the patient is on the verge of a serological and perhaps clinical relapse. On the other hand, as I have already pointed out, it may be a non-specific phenomenon and due to some disease other than syphilis. Following some work done by Witebsky, ${ }^{5}$ recently I have tested a number of such sera by fortifying them with an extract of known positive sera and re-submitting them to the routine Wassermann reaction. It is claimed that in these circumstances a specific \pm will be converted to a frank positive, whilst a non-specific will remain \pm or even become negative ; the tests I have carried out tend to support this theory. In such circumstances Dr. Wyler ${ }^{6}$ recommends the use of larger amounts of patient's serum - two, three, four or even five times as much as usually employed-and obtains similar results. Whilst on the subject of borderline reactions, may I call your attention to a "wobbling" serum reaction? I expect you have all come across cases which give varying reactions, sometimes negative, sometimes positive, sometimes doubtful. Take my advice and regard such cases with the gravest suspicion. In my experience such cases do not react well to treatment, and I recommend you to give the most guarded prognosis.

Now as to the cerebro-spinal fluid. What are the indications and optimum times for the examination of 


\section{BRITISH JOURNAL OF VENEREAL DISEASES}

this? Unfortunately the objections of the patient and the possibility of more or less severe reactions set a limit to the frequency with which this can be done, though with the use of Colonel Harrison's fine needle these latter have been reduced to negligible proportions. Stokes thinks the fluid should be examined during the first course of treatment in early cases. Seeing that the result is not likely to affect the treatment, it would seem that the examination might coincide with the termination of treatment in such cases, or as a matter of convenience it might be done any time during the two years of observation. There is a good deal to be said for fixing it at the end of two years following completion of treatment, that is to say, three to four years from the date of infection; if the fluid is negative then it is extremely unlikely to become positive subsequently. In any circumstances no case of syphilis should be passed as cured without a negative cerebro-spinal fluid.

Other conditions which indicate lumbar puncture are : (I) Persistent positive serum tests, and (2) any sign or symptom indicating central nervous system involvement. In cerebro-spinal syphilis, tabes and G.P.I. an examination is usually required for confirmation of the diagnosis ; subsequent examinations are needed to observe the effect of treatment. These may profitably be done annually, or more often if thought necessary. Lumbar puncture for purposes of treatment does not come within the scope of this paper. For the examination of the spinal fluid I recommend the Wassermann reaction (using two neat volumes of fluid or ten times as much as in the corresponding serum test), the cell count and a test for globulin. A colloidal reaction if you like, though it hardly seems to me to give much information except in tabes and G.P.I., where I admit it may be of considerable value. You may choose between the Lange or colloidal gold, the colloidal mastic or the colloidal benzoin. I have rather a preference for the mastic, more especially as the gold is so difficult to make. I doubt if any of the flocculation tests presents any advantage over the Wassermann reaction in this connection. A positive Wassermann reaction on the fluid is even more specific than on the serum-false positive reactions must be very rare. Less than five cells may be regarded as negative (Stokes says three), five to ten as doubtful, and more than ten as 


\section{THE VALUE OF SERUM REACTIONS}

pathological. The presence of more than a trace of globulin should be regarded with grave suspicion.

General paresis, untreated, gives practically roo per cent. positives with all four tests. In tabes the reactions are usually not so strongly or uniformly positive ; perhaps 60 to 70 per cent. positive Wassermann reactions is as much as can be expected. The number of cells is usually less than in G.P.I. and the amount of globulin less also. It is in the quiescent cases that one must expect a certain proportion of negative results. Cerebro-spinal syphilis gives a very high percentage of positive Wassermann reactions-usually a very large number of cells, often running into hundreds per cubic millimetre, and a wellmarked globulin reaction.

The colloidal reactions are often useful in distinguishing between the various forms of syphilis of the central nervous system. A typical paretic curve shows precipitation in the left-hand tubes, which contain the lower dilutions of fluid, tailing off towards the right-hand end. The luetic curve usually shows partial precipitation in the tubes containing the medium dilutions: I do not believe in the so-called tabetic curve. Under treatment the colloidal reactions and cells usually clear up first, globulin second, and the Wassermann reaction last. Cases showing a positive Wassermann reaction in the higher dilutions of the fluid and a more or less typical paretic colloidal reaction are the most resistant to treatment.

Much of what has been said of the Wassermann applies equally to the various flocculation tests. The best of these, in my opinion, are the Kahn, Müller-Ballungs (M.B.R.II.), and Meinicke-Klärungs (M.K.R.). I prefer the first named because it is simpler to carry out and the result is obtainable in a few minutes, though I admit the reading sometimes presents some difficulty, especially to the novice. The relative value of the Wassermann reaction and Kahn has been the subject of much discussion, but I do not think it is a matter of great importance, since my advice and practice is to use both. Few will deny that the Kahn is the more sensitive in treated cases-I stress the word " treated." In untreated cases - at any rate in early primary ones-I do not believe there is anything to choose between the two tests. A year or two ago I took a considerable number of primary cases which reported at the St. Thomas's Hospital clinic and submitted their sera 


\section{BRITISH JOURNAL OF VENEREAL DISEASES}

to both tests. The result showed a remarkably close agreement. In the case of cerebro-spinal fluids I think the Wassermann reaction has the advantage, because in point of specificity and sensitivity it is practically the equal of the Kahn and it is far less difficult and laborious to carry out ; moreover, the Kahn fails if the specimen is not a clear one. There is one other flocculation reaction which I should like to bring to your notice, and that is the Sigma or Dreyer-Ward test. In my experience this is the only satisfactory quantitative test we have. Many pathologists carry out a quantitative Wassermann reaction by diluting the patient's serum, say, I in 5, I in Io, I in 20 , and so on. I mistrust the results of these tests, and I have done many myself. A simple experiment will explain why. Take a number of different complements and titrate them out in parallel in the presence of antigen plus saline and in the presence of antigen plus the same amount of a known negative serum as one uses in the Wassermann reaction. You will find frequently that the end point in the two titrations differs by quite a lot-the row of tubes containing the serum showing a much higher titre of complement than the one containing saline-in other words, the serum has protected the complement against the action of the antigen. Now if you perform a Wassermann reaction with increasing dilutions of your patient's serum you will have a progressively diminishing amount of serum present and your end point may be much higher than it should be. We now take advantage of this fact in doing quantitative complement-fixation tests for gonorrhœa, since we have at present no reliable flocculation tests to control the complement-fixation one.

To return to the Sigma test, which records results in units. We are all constantly running up against the Wassermann-fast latent case who keeps on giving a positive reaction no matter how many courses of treatment we give him. He has no physical signs, and in fact we have no means of knowing whether our treatment is having any effect or not ; moreover, the patient is apt to get dispirited and less and less inclined to persevere with treatment. A Sigma test on such a case shows, say, fifty units before treatment ; twenty after the first course ; twelve after the second; five after the third, and two after the fourth. Obviously treatment has accomplished something ; moreover, the patient has got something to I86 


\section{THE VALUE OF SERUM REACTIONS}

"bite on" so to speak, and is encouraged thereby. When your Sigma reaction "sticks," i.e., when you cannot get it any lower, usually you are safe in discontinuing active treatment and watching your case.

From what I have said you will realise that a single Sigma reaction is not of great value; it is a series of these tests which is often so useful. Personally I do not regard the Sigma test as practical politics for routine diagnostic use. It requires a relatively large amount of patient's serum, is laborious to set up, and the result is not available till the next day.

Now I propose to leave the subject of syphilis and turn to that of gonorrhoea. Few, I suppose, will deny that the complement-fixation test has proved its value in the diagnosis and management of gonorrhœa and has come to stay. I do not say that the test is perfect or incapable of improvement, but I do say that it is a very useful weapon in our armamentarium and one that none of us can afford to neglect; not nearly enough use is made of it. If all gonorrhœal patients had their sera tested before commencing treatment, and thereafter monthly, a great deal of valuable information would be obtained. In my opinion the test bears much the same relation to gonorrhœa as the Wassermann reaction does to syphilis. Tending to become positive towards the end of the first week of the disease, the percentage of reactions increases week by week, in an untreated case, till by about the fourth to fifth week one may expect it to be in the neighbourhood of roo. As, under the effect of treatment, the gonococcus tends to die out, so the reaction diminishes till after a variable time following cure it gradually weakens and becomes negative. Rarely one meets a case in which antibody production appears to be almost negligible, and in such a one the reaction remains negative throughout.

The complement-fixation is a measure of anti-body production; therefore, assuming that the tissues are reacting, the greater the absorption of toxin the greater will be the formation of anti-body. It will be seen, therefore, that so long as the infection is mild and limited in extent the reaction is likely to be negative, whereas when the infection spreads to the deeper tissues the reaction will become positive. This is in accord with clinical observation-a simple anterior urethritis usually 


\section{BRITISH JOURNAL OF VENEREAL DISEASES}

gives a negative reaction-but when a posterior urethritis develops in the male or the infection spreads to the cervix uteri in the female a positive reaction is to be expected. This is especially true of complications, which give nearly roo per cent. of positives. It is here that the reaction is most valuable-the cause of an obscure arthritis or salpingitis often being determined by the result of the test ; I would be prepared to lay heavy odds that a case of sub-acute arthritis giving a negative reaction is not gonorrhœal in origin. I have not much experience of vulvo-vaginitis; but my impression is that when the infection is limited to the vagina the reaction is negative, when it reaches the cervix the reaction becomes positive. As regards specificity the reaction ranks almost with the Wassermann reaction. A positive one means gonorrhœa present or recently cured in from 90 to Ioo per cent. of cases. To what extent crossfixation takes place with meningococcal catarrhalis and flavus infections I am not prepared to say. Work done on this subject by Arkwright, Oliver and Price has not settled the question. Oliver thinks that $M$. catarrhalis is a danger, but Price's experiments are against this. Much more work requires to be done before the matter can be cleared up. In view of the prevalence of catarrhal infections, especially in the winter months, the possibility must always be considered in the presence of an unexpected positive reaction.

Speaking generally, a positive reaction indicates active gonorrhœa, whilst a negative implies that a patient either has not got gonorrhœa, or if he has that the infection is not deep or that, rarely, he is not reacting to it. Naturally, of course, the result of the test must always be considered in conjunction with the history and clinical findings. Vaccines will usually give rise to a positive reaction even in a normal individual, but according to Price this falls to negative six weeks after the vaccine is discontinued; in a case suffering from gonorrhœa they may cause an intensification of the reaction.

\section{TEST OF Cure}

A negative reaction in a patient clinically free is presumptive evidence, but not proof, of cure, especially if the reaction has previously been positive. How soon 


\section{THE VALUE OF SERUM REACTIONS}

the reaction becomes negative following the disappearance of the last gonococcus I am not prepared to say ; opinions differ-probably it varies a good deal. In order to establish the fact of cure with a reasonable degree of certainty the reaction as well as microscopical and cultural tests should be negative three months after cessation of treatment, and for purposes of marriage I like to confirm these at the end of a year. What about the patient who gives a persistent positive following apparent cure? Are we to assume, as has been done by some, that this invariably means that the patient is harbouring living gonococci in his tissues, is potentially infectious and should be debarred indefinitely from marriage ? I confess I find it hard to believe. I have in mind patients whose reactions have remained strongly positive for years in whom, though I searched over and under, I could find no trace of the gonococcus. What are we to say when such cases fail to infect their wives and even produce children, proving that they have emptied their seminal vesicles, the deepest and most inaccessible lair of the gonococcus? We can only say "Not proven." I confess I can offer no satisfactory explanation. I can only say that in my experience these are frequently cases who have suffered from some complication, and perhaps the gonococcus if present is so sealed in that it cannot escape. Alternatively it may be a case of cross-fixation ; at any rate this should be kept in mind. In these cases it is worth while (I) to test out the serum against catarrhalis and flavus antigens, and (2) to carry out a number of quantitative tests to see if the reaction is increasing, decreasing or remaining stationary.

There are those who belittle the value of the complement-fixation reaction, but the man who undertakes the management of gonorrhœa without its aid is handicapping himself severely in a most difficult undertaking.

Here, may I offer a word of advice? Always have a Wassermann reaction carried out on your gonorrhœa cases ; many a patient contracts syphilis at the same time, and this may not show itself, especially in the female. I would like to conclude with a truism. Serum tests, like the rest of clinical pathology, should be your servant and not your master; what I have said to-night is subject to that proviso.

If my remarks have been in the nature of instruction 


\section{BRITISH JOURNAL OF VENEREAL DISEASES}

of an elderly female relative in swallowing the contents of oval bodies laid by birds, I crave your indulgence. If, on the other hand, anything I have said has been of help to some of those who have listened so patiently, I shall feel amply repaid for any little trouble I have taken in preparing this paper.

Ladies and Gentlemen, I thank you.

\section{REFERENCES}

(I) Amies, C. R. : Malaya Med. Jour., Vol. 4., No. 4, pp. I29-I32.

(2) Lloyd, R. B., Muir, E., and Mitra, G. C.: Ind. Jour. Med. Res., Vol. 14., No. 3, p. 667.

(3) Kolmer, J. A. : "Serum Diagnosis by Complement Fixation," I929, P. 473 .

(4) WARTHIN, A. S. : Brit. Med. Jour., I929, Vol. ii., p. 236.

(5) Witebsкy, E. : Ztschr.f. Immun. u. Exp. Therap., I933, Vol. 80, p. 323 .

(6) Wyler, E. J. : Ministry of Health Special Report, No. 67. 